Article

\title{
International System of Units (SI) Traceable Noise-Equivalent Power and Responsivity Characterization of Continuous Wave ErAs:InGaAs Photoconductive Terahertz Detectors
}

\author{
Anuar de Jesus Fernandez Olvera ${ }^{1, *}$, Axel Roggenbuck ${ }^{2}$, Katja Dutzi ${ }^{2}$, Nico Vieweg ${ }^{2}$, Hong Lu ${ }^{3}$, \\ Arthur C. Gossard ${ }^{4}$ and Sascha Preu ${ }^{1}$ \\ 1 Terahertz Devices and Systems, Department of Electrical Engineering and Information Technology, \\ Technische Universität Darmstadt, Merckstr. 25, 64283 Darmstadt, Germany; preu@imp.tu-darmstadt.de \\ 2 TOPTICA Photonics AG, Lochhamer Schlag 19, D-82166 Gräfelfing, Germany; \\ Axel.Roggenbuck@toptica.com (A.R.); Katja.Dutzi@toptica.com (K.D.); nico.vieweg@toptica.com (N.V.) \\ 3 College of Engineering and Applied Sciences, Nanjing University, Hankou Road 22, Nanjing 210093, China; \\ hlu@nju.edu.cn \\ 4 Materials Department, University of California Santa Barbara, Santa Barbara, CA 93106, USA \\ gossard@engineering.ucsb.edu \\ * Correspondence: fernandez@imp.tu-darmstadt.de; Tel.: +49-6151-16-28489
}

Received: 14 December 2018; Accepted: 7 February 2019; Published: 13 February 2019

\begin{abstract}
A theoretical model for the responsivity and noise-equivalent power (NEP) of photoconductive antennas (PCAs) as coherent, homodyne $\mathrm{THz}$ detectors is presented. The model is validated by comparison to experimental values obtained for two ErAs:InGaAs PCAs. The responsivity and NEP were obtained from the measured rectified current, the current noise floor in the PCAs, and the incoming $\mathrm{THz}$ power for the same conditions. Since the $\mathrm{THz}$ power measurements are performed with a pyroelectric detector calibrated by the National Metrology Institute of Germany (PTB), the experimentally obtained values are directly traceable to the International System of Units (SI) for the described conditions. The agreement between the presented model and the experimental results is excellent using only one fitting parameter. A very low NEP of $1.8 \mathrm{fW} / \mathrm{Hz}$ at $188.8 \mathrm{GHz}$ is obtained at room temperature.
\end{abstract}

Keywords: Coherent THz detectors; photoconductive antennas; responsivity; NEP; homodyne detection

\section{Introduction}

Photoconductive antennas are an established technology for $\mathrm{THz}$ detection in commercial continuous wave (CW) spectrometers [1]. Their use in applications other than spectroscopy, such as communication [2,3] and non-destructive testing [4-6], has been fostered by the introduction of photoconductive antennas (PCAs) compatible with $1550 \mathrm{~nm}$ telecom laser technology [7,8]. These laser systems benefit from the large availability of inexpensive, powerful optical components of the telecom industry.

A full characterization of the PCAs performance as THz detectors is still difficult. In most cases, only the system dynamic range is specified $[9,10]$, which is a relative figure of merit, composed of the source performance and receiver performance. International System of Units (SI) traceable noise floors and power levels are seldomly specified. To the knowledge of the authors, the only SI traceable characterization has been performed on pulsed PCAs in [11,12].

In this work, we experimentally characterize the noise-equivalent power (NEP) and the responsivity of two PCA homodyne $\mathrm{CW}$ THz detectors, and develop a theoretical model for them. 
The experimental characterization is performed by measuring the detected current and the noise floor of the PCAs when a THz PIN diode is used as an emitter; then, the PCAs are replaced by a pyroelectric detector, and the emitted power is measured under exactly the same conditions. Since the THz power is measured with a pyroelectric detector calibrated by the National Metrology Institute of Germany (PTB) [13], the figures of merit obtained under these conditions are directly traceable to SI units. The characterization incorporates the DC properties of the detectors as well as a comparison with the DC properties of a third detector with similar properties. The first two characterized $\mathrm{THz}$ detectors are ErAs:InGaAs PCAs with a $10 \mu \mathrm{m} \times 10 \mu \mathrm{m}$ active area covered by a finger electrode structure which is coupled to a self-complementary logarithmic-periodic antenna. The photoconductive material consists of a periodic structure of alternating layers of $10 \mathrm{~nm}$ of InGaAs and Be-delta-doped 0.8 monolayers of ErAs repeated 90 times. The third detector, used only for the DC comparison, is identical to the first two but instead of being Be-doped, it was C-doped, as described in [14]. All three detectors were designed and processed at Technische Universität Darmstadt with material grown at the University of California, Santa Barbara solely for research purposes.

The experimental characterization is performed for the frequency range between $100 \mathrm{GHz}$ and $1 \mathrm{THz}$. The obtained results are compared with theoretical predictions. The theoretical model is elaborated in Section 2. The setup for the experimental characterization and the conditions in which it was performed are described in Section 3. The comparison between the experimental and the theoretical results is presented in Section 4. Brief concluding remarks are given in Section 5.

\section{Theoretical Model for the NEP and Responsivity of PCAs}

A PCA coherent THz detector with a finger-electrode structure as shown in Figure 1a generates a rectified current

$$
I_{r}(f, t)=\int_{A} j(\vec{r}, f, t) d A
$$

where $f$ is the THz frequency (frequency difference between the lasers driving the PCA), $t$ time, $j$ the current density, $\vec{r}$ the spatial coordinate in the cross section area, and $A=d_{S} L_{T}$ the meander-like cross section area between the finger electrodes on the absorbing photoconductive layer with thickness $d_{S}$ and meander length $L_{T}$.

The total length can be approximated to $L_{T} \approx(N-1) \cdot\left(L_{F}+w_{e}+w_{G}\right)$ with $N$ the total number of fingers, $L_{F}$ the finger length, $w_{e}$ the finger width, and $w_{G}$ the photoconductive gap size, as shown in Figure 1a.

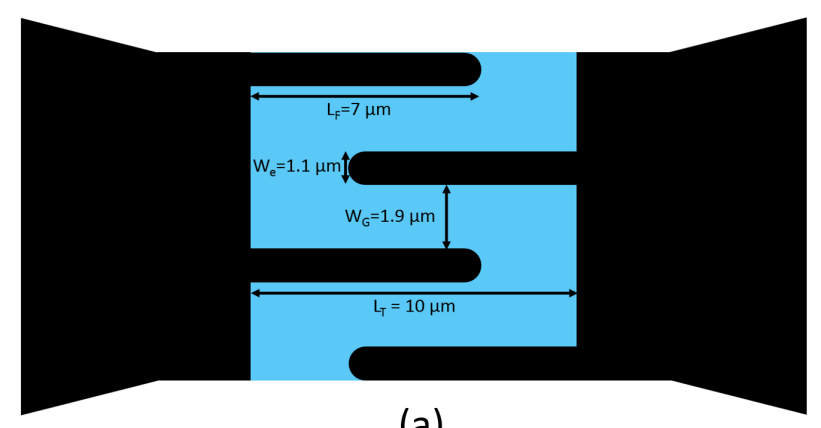

(a)

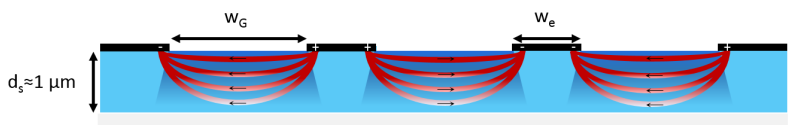

(b)

Figure 1. Geometric layout of the finger electrode (black) structure on the photoconductive material (blue) with geometric variables as defined in the text: (a) Top view (b) Cross-sectional view (red lines depict the field lines across the gap). 
The current density generated by holes or electrons has been derived in [15], Equation (9), to

$$
j(z, f, t)=\frac{e \alpha P_{L}(z) v(z) \tau_{\text {rec }}}{L w_{G} h v_{L}} \cdot\left(\frac{\cos (\omega t)}{1+i \omega \tau_{\text {rec }}}+1\right),
$$

with $\omega=2 \pi f, P_{L}(z)$ the laser power at depth $\mathrm{z}, \alpha$ the absorption coefficient, $v_{L}$ the frequency of the laser driving the PCA, $v(z)$ the average carrier velocity at depth $z, \tau_{\text {rec }}$ the carrier lifetime (assumed to be the same for holes and electrons) and $\eta_{\tau}^{I}=\left(1+i \omega \tau_{\text {rec }}\right)^{-1}$ the lifetime roll-off for the current. The absolute-square of $\left|\eta_{\tau}^{I}\right|^{2}=\eta_{\tau}$ is the well known lifetime roll-off for the THz power. The term $\frac{v(z) \tau_{\text {rec }}}{w_{G}}$ can be understood as the photoconductive gain which is, as we will show, linearly dependent on the received $\mathrm{THz}$ field.

The average carrier velocity $v=n^{-1} \int v(z) d n(z)$ originates from the THz bias $U_{T H z}$ produced in the antenna when $\mathrm{THz}$ power falls on it, with $n$ being the total amount of generated carriers and $n(z)$ the amount of carriers at depth $z$. Its value, weighted by the amount of carriers, is given by $v=\mu E_{g a p}$, where $\mu$ is the respective carrier mobility and

$$
E_{\text {gap }}(f, t)=\frac{\eta_{\text {bias }} \sqrt{\eta_{R C}(f)} U_{T H z} \cos (\omega t)}{w_{G}}
$$

is the (effective) $\mathrm{THz}$ field in the gap which is proportional to the $\mathrm{THz}$ bias delivered by the antenna that drops along the gap width, $w_{G}$. The efficiency $\eta_{\text {bias }}<1$ accounts for the inhomogeneity of the field between the electrodes (as shown by the field lines in Figure 1b), and for the variation of carrier density as a function of penetration depth with $z$ as a weighting factor. The term $\left|\eta_{R C}(f)\right|=\left|1+i \omega Z_{A}(f) C\right|^{-2}$ is the power RC roll-off, where $Z_{A}(f)$ is the antenna impedance, and $C$ is the device capacitance, as shown in the equivalent circuit of the PCA in Figure 2. The effect of photoconductor resistance $R_{P C}$ is usually negligible for the RC roll-off under CW operation conditions, where typically $R_{P C} \gg\left|\frac{i}{\omega C}\right|$ and $R_{P C} \gg\left|Z_{A}(f)\right|$. For the PCAs analyzed here and the frequency range of interest, electromagnetic (EM) simulations show that $\left|Z_{A}(f)\right|$ achieves a maximum of $125.75 \Omega$ and that $C$ equals $3.7 \mathrm{fF}$, resulting in a maximum of $442 \Omega$ for $\left|\frac{i}{\omega C}\right|$. The measured value of $R_{P C}$ is between $12.8 \mathrm{k} \Omega$ and $16.66 \mathrm{k} \Omega$. Hence, $R_{P C}$ is at least 30 times higher than $\left|\frac{i}{\omega C}\right|$ and at least 100 times higher than $\left|Z_{A}(f)\right|$, thus, its effect can be considered negligible.

Since the effective mass of (heavy) holes $m^{*}$ is about nine times larger than that of electrons in $\mathrm{In}_{0.53} \mathrm{Ga}_{0.47} \mathrm{As}$, the mobility of holes, $\mu \sim \tau_{p} / m^{*}$, is estimated to be about nine times smaller than that of electrons (assuming similar momentum scattering times $\tau_{p}$ for both electrons and holes). Therefore, holes play only a minor role in the current generation mechanism, and only electrons are taken into account. With that consideration, the substitution of all terms into Equation (1) yields

$$
I_{r}(f, t)=\frac{\eta_{o p t} \eta_{\text {bias }} I_{i d} \mu \tau_{r e c}}{w_{G}^{2}} \cdot\left(1+\cos (2 \pi f t) \sqrt{\eta_{\tau}(f) \eta_{R C}(f)}\right) \cdot U_{T H z} \cos (2 \pi f t+\varphi) .
$$

The phase difference $\varphi$ accounts for the optical path length difference between source and receiver; here, we are only interested in peak values, hence, we set $\varphi=0 . I_{i d}=\frac{e P_{L}}{h v_{L}}$ is the ideal photocurrent amplitude for the total incident laser power $P_{L}$. The optical efficiency $\eta_{\text {opt }}$ takes into account that not all the incident laser power is absorbed within the photoconductor of thickness $d_{s}$.

The THz bias is related to the incoming THz power $P_{T H z}(f)$ by $U_{T H z}(f)=\sqrt{2 \eta_{T H z} P_{T H z}(f) R_{A}(f)}$ with $R_{A}(f)$ the real part of $Z_{A}(f)$ and $\eta_{T H z}$ the coupling efficiency of the THz beam to the antenna, which takes into account the mismatch of the $\mathrm{THz}$ spot size to the effective antenna cross section and the silicon lens reflections. The value of $U_{T H z}$ in the equivalent circuit is the peak value of voltage source that would re-radiate all the incoming $P_{T H z}(f)$ if the output terminals of the antenna were left open (i.e., the Thevenin equivalent open-circuit voltage source) as explained in [16]. A generalized 
formulation for broadband excitation would require substituting the powers by spectral densities. For the case of broadband pulsed excitation, the derivation for emitted power can be found in ref. [17].
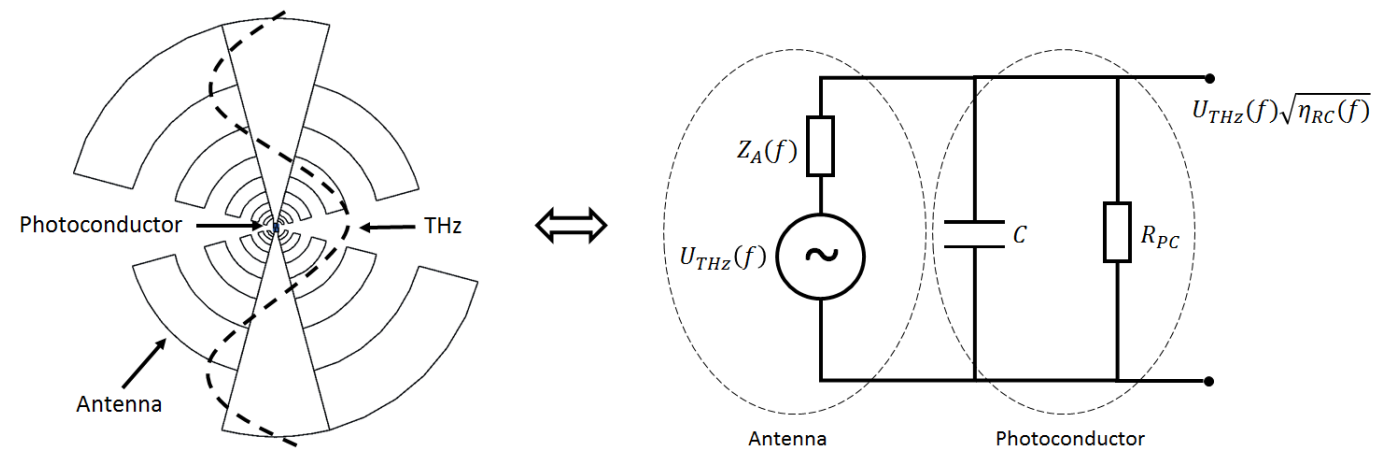

Figure 2. Equivalent circuit of the receiving antenna including the incoming $\mathrm{THz}$ wave with power $P_{T H z}(f)$, the antenna and the photoconductor. The value of $U_{T H z}(f)$ is the Thevenin equivalent open-circuit voltage source, as defined in [16].

The detected current as a function of the $\mathrm{THz}$ power can then be written as

$$
I_{r}(f)=<I_{r}(f, t)>=\frac{1}{2} \cdot \frac{\eta_{o p t} \eta_{\text {bias }} I_{i d} \mu \tau_{\text {rec }}}{w_{G}^{2}} \cdot \sqrt{2 \eta_{\tau}(f) \eta_{R C}(f) \eta_{T H z} P_{T H z}(f) R_{A}(f)} .
$$

where $<$. $>$ denotes the time average, i.e., the DC component.

The unknowns $\eta_{\text {opt }}$ and $\eta_{\text {bias }}$ can be determined by a measurement of the DC resistance, since Equation (4) is also valid at DC if one substitutes the THz bias $U_{T H z} \cos (\omega t+\varphi)$ by the DC bias $U_{D C}$. This results in $\left.<U_{D C}\left[1+\sqrt{\eta_{\tau}(f) \eta_{R C}(f)} \cos (2 \pi f t)\right]\right\rangle=U_{D C}$. Equation (4) then becomes

$$
I_{r}^{D C}=\frac{\eta_{o p t} \eta_{b i a s} I_{i d} \mu \tau_{r e c} U_{D C}}{w_{G}^{2}}=\frac{U_{D C}}{R_{P C}},
$$

with

$$
R_{P C}=\left(\frac{\eta_{\text {opt }} \eta_{\text {bias }} I_{\text {id }} \mu \tau_{\text {rec }}}{w_{G}^{2}}\right)^{-1}
$$

being the DC resistance of the PCA under illumination. Ultimately, we can define the $\mathrm{THz}$ power responsivity $S(f)$ as

$$
I_{r}^{2}(f)=S(f) \cdot P_{T H z}(f),
$$

with

$$
S(f)=\frac{1}{2} \eta_{\tau}(f) \eta_{R C}(f) \eta_{T H z} R_{A}(f) \cdot\left(\frac{\eta_{\text {opt }} \eta_{\text {bias }} I_{\text {id }} \mu \tau_{r e c}}{w_{G}^{2}}\right)^{2}=\frac{1}{2} \eta_{\tau}(f) \eta_{R C}(f) \eta_{T H z} \cdot \frac{R_{A}(f)}{R_{P C}^{2}} .
$$

Except for $\eta_{T H z}$, which is alignment-dependent, all the parameters in Equation (9) can be obtained from EM simulations $\left(\eta_{R C}(f)\right)$ or from previous measurements $\left(\eta_{\tau}(f)\right)$, as it will be shown in Section 4. This, however, assumes that the $\mathrm{DC}$ and $\mathrm{THz}$ material parameters of the photoconductor are identical, which may not generally be the case. As an example, the DC mobility as obtained from Hall measurements is well suited to determine the DC resistance of the sample. However, under $\mathrm{THz}$ conditions, the carriers are only inadequately described by a constant mobility which describes the constant average velocity, $v_{a v}=\mu E$, for a carrier in an electric field. On the one hand, photocarriers are generated at rest and then accelerated to $v_{a v}$, generally leading to smaller average velocities as predicted by the DC mobility; further, the acceleration period may have influence on the frequency response under pulsed operation. For CW, however, non-harmonic and higher harmonic contributions 
average out, allowing to describe the carrier velocity by an average value. On the other hand, intrinsic samples with few scattering centers can even show a velocity overshoot [18]; but it is generally accepted that a velocity overshoot is not expected for photoconductors due to scattering with trap states and the short carrier lifetime. We therefore expect that the mean velocity of photogenerated electrons on the sub-ps time scale, relevant for $\mathrm{THz}$ rectification, is smaller than the average DC velocity $v_{a v}$ relevant for DC measurements. An exact determination of the carrier motion on a $\mathrm{THz}$ time scale requires further pump-probe measurements on the sub-ps time scale that go beyond the scope of this paper. Hence, $\eta_{T H z}$ is the only fitting parameter in our model of the responsivity. In general, $\eta_{T H z}$ might be a function of frequency, but for the frequency range of interest, between $100 \mathrm{GHz}$ and $1 \mathrm{THz}, \eta_{T \mathrm{~Hz}}$ is indeed fairly frequency-independent. The reason is that the effective receiving area in the PCA detector given by the broadband logarithmic-periodic antenna and the diffraction-limited spot size of the incident $\mathrm{THz}$ power scale with frequency in the same manner (area $\sim f^{-2}$ ). Further the emitter and the detector use self-complementary antennas. Even if there is a mismatch between the effective receiving area and the $\mathrm{THz}$ spot size, the mismatch will remain the same through the whole frequency range of interest due the frequency-independent properties of self-complementary antennas. Only at frequencies below $150 \mathrm{GHz}$, the $\mathrm{THz}$ spot is diffraction-limited by the finite aperture of the silicon lens attached to the detectors. This results in further Airy focusing and in a slight shift in the focal point of the THz spot, reducing the coupling efficiency. Other than that, the reflections at the surface of the silicon lens (due to the mismatch between the refractive index of silicon and air) remain constant for the whole frequency range.

Considering the differences between AC and DC performance, a more appropriate formulation of Equation (9) for CW operation is

$$
S(f)=\frac{1}{2} \eta_{\tau}(f) \eta_{R C}(f) \eta_{T H z} \frac{\mu_{T H z}^{2}}{\mu_{D C}^{2}} \cdot \frac{R_{A}(f)}{R_{P C}^{2}} .
$$

While the THz mobility $\mu_{T H z}$ is unkonwn, the DC mobility $\mu_{D C}$ can be obtained from Hall measurements. Given that we cannot discriminate experimentally between inefficient coupling and differences in the mobility, we define an effective THz efficiency $\eta_{T H z}^{e f f}=\eta_{T H z} \frac{\mu_{T H z}^{2}}{\mu_{D C}^{2}}$.

Finally, we derive an equation for the NEP, defined as the amount of power required to have a signal-to-noise ratio of one. The ideal NEP can then be expressed as

$$
N E P_{T H z}(f)=\frac{I_{\text {noise }}^{2}(f)}{S(f)}
$$

where $N E P_{T H z}(f)$ is the THz NEP (with units $\mathrm{W} / \mathrm{Hz}$ ) and $I_{\text {noise }}=I_{\text {therm }}=\sqrt{4 k_{B} T / R_{P C}}$ is the thermally limited noise, being the lower limit for the noise current density if further noise sources, such as undesired incoupling of stray fields or amplifier noise are absent.

Substituting Equation (9) and using $I_{\text {noise }}=I_{\text {therm }}$ into Equation (11) yields

$$
N E P_{T H z}(f)=\frac{8 k_{B} T}{\eta_{T H z}^{e f f} \eta_{\tau}(f) \eta_{R C}(f)} \cdot \frac{R_{P C}}{R_{A}(f)} .
$$

We remark that the derivation so far assumed that the photoconductor resistance, $R_{P C}$, is much larger than the radiation resistance of the antenna, $\left|Z_{A}(f)\right|$, i.e., it is mismatched. This is the usual case for $\mathrm{CW}$ operation. For pulsed operation, however, $R_{P C}$ may become comparable to or even smaller than $\left|Z_{A}(f)\right|$, at least for short times. In this case, the $N E P_{T H z}$ in Equation (12) needs to be modified by reevaluation of the equivalent circuit in Figure 2 [12]. As an example, in the extreme case of $R_{P C} \ll\left|Z_{A}(f)\right|$, the THz bias generated by the antenna drops almost completely at $Z_{A}(f)$ (i.e., it is re-radiated) and only negligible bias drops at the photoconductor. The device stops working efficiently. 


\section{Experimental Setup}

Two THz setups, both using the TeraBeam 1550 system from Toptica Photonics, were used for the experimental determination of the NEP and responsivity of the two ErAs:InGaAs PCAs, henceforth PCA A and B (Figure 3).

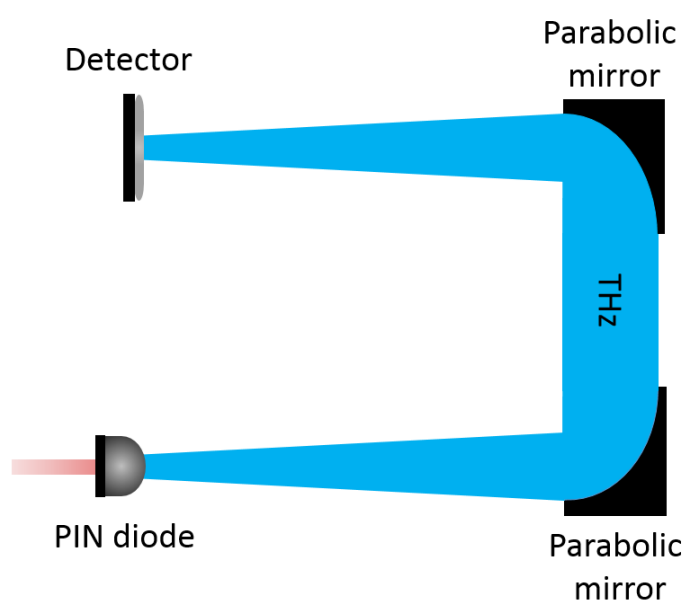

Figure 3. Experimental setup for the measurements. For THz power measurement, the detector was a pyroelectric device, calibrated by the PTB. For the THz current measurements, it was replaced by the PCAs.

First, the power of a PIN diode, used as the THz emitter to characterize both PCAs, was measured using a pyroelectric detector calibrated by the PTB. To do so, a lock-in amplifier was connected to the detector in order to read out the root mean square (RMS) value of the detected voltage. A second calibration was required in order to transform the RMS value (measured by the lock-in amplifier) into a peak-to-peak value (measured by an oscilloscope at the PTB). The PTB calibration of the detector was performed with a maximum error of $5 \%$, and it allowed to measure a minimum power of $1 \mu \mathrm{W}$. The calibration of the lock-in amplifier RMS value with respect to the oscilloscope peak-to-peak value featured an error of $2 \%$ (after a total of 10 measurements).

The setup used for the power measurements employed two parabolic mirrors (with a diameter of $5.08 \mathrm{~cm}$, and focal length of $7.62 \mathrm{~cm}$ ) to collimate and focus the $\mathrm{THz}$ radiation from the $\mathrm{THz} \mathrm{PIN}$ diode. The pyroelectric detector was placed at $5.5 \mathrm{~cm}$ from the focusing mirror, the same distance at which the PCA detectors were placed. The THz PIN diode was biased with a $15.5 \mathrm{~Hz}$ square signal to produce a slow $\mathrm{THz}$ ON/OFF modulation as required by the pyroelectric detector due to its slow response time. In order to completely shut down the $\mathrm{THz}$ emission, a forward bias of $0.5 \mathrm{~V}$ was always applied during the OFF part of the modulation cycle. For the different $\mathrm{THz}$ powers required to determine $\eta_{T H z}^{\text {eff }}$, different reverse biases between $-0.2 \mathrm{~V}$ and $-1.1 \mathrm{~V}$ were applied during the ON part of the modulation cycle. For the experimental characterization of the NEP and the responsivity, the reverse bias was kept constant at $-1.1 \mathrm{~V}$ during the ON part. The optical power arriving at the PIN diode was $26 \mathrm{~mW} \pm 0.7 \mathrm{~mW}$ during the characterization of PCA A, and $22 \mathrm{~mW} \pm 0.3 \mathrm{~mW}$ during the characterization of the PCA B.

Second, the pyroelectric detector was replaced by the PCAs. The modulation frequency was increased to $1983 \mathrm{~Hz}$ in order to reduce $1 / f$ noise. We verified that the current amplitude detected by the PCA with a modulation frequency of $15.5 \mathrm{~Hz}$ frequency was identical to that detected with $1983 \mathrm{~Hz}$, confirming that the THz power is not affected by modulation speed of the PIN diode. The PCAs were connected to a transimpedance amplifier (TIA) with a gain of $3.3 \times 10^{5} \mathrm{~V} / \mathrm{A}$, and then to a lock-in amplifier. The laser power from the TeraBeam 1550 arriving at PCA A during its characterization was $25.3 \mathrm{~mW} \pm 0.6 \mathrm{~mW}$, while the laser power arriving at PCA B during its characterization was 
$24.7 \mathrm{~mW} \pm 0.9 \mathrm{~mW}$. This results in an uncertainty of $3.6 \%$ in the measured laser power arriving at the PCAs, and hence, in the NEP.

The laser power at the THz PIN diode and the PCA detectors was defined by the output power of the DFB lasers of the TeraBeam 1550. The laser power arriving at all the devices was measured with an optical power meter after the $\mathrm{THz}$ measurements were performed. The differences in the measured laser power between the characterization of PCA A and PCA B were caused by the use of different fibers. Variations in the measured laser power arriving at the PIN diode result in differences of the $\mathrm{THz}$ power. These differences are calibrated by $\mathrm{THz}$ power measurement with the PTB-calibrated detector and therefore do not affect the NEP measurement. Variations in the measured laser power arriving at the detectors, however, may alter the NEP and the responsivity. As discussed in Ref. [14], the NEP saturates at high laser powers, $P_{L}$, and then variations of $P_{L}$ have no influence on the NEP. For low laser powers, the NEP is proportional to $P_{L}^{-1}$ according to Equation (12), resulting in a (worst case) NEP uncertainty of $3.6 \%$. The geometric mean of all known uncertainties in the NEP then accounts to $6.5 \%$. The responsivity, according to Equation (9) is proportional to $P_{L^{\prime}}^{2}$ thus, its uncertainty due to the errors in the laser power measurements is $7.2 \%$. The geometric mean of all known uncertainties in the responsivity accounts then to $9 \%$.

Remaining uncertainties are alignment errors. The PTB-calibrated power detector features an area much larger than the $\mathrm{THz}$ spot size, easily capturing all incoming $\mathrm{THz}$ power, while the PCA, with an effective receiving area in the range of the $\mathrm{THz}$ spot size, requires excellent alignment to achieve the same. The remaining uncertainty is therefore the alignment error of the PCA to the THz spot. As there are no means to access this quantity accurately, the alignment uncertainty is swallowed in the only fitting parameter $\eta_{T H z}^{e f f}$. All values presented in the following are therefore extrinsic. The intrinsic detector NEP may be much lower and the responsivity much higher than the presented values, leaving room for future improvements of the detector design.

\section{Results}

In order to compare experimental results to the theoretical model, i.e., the DC resistance (Equation (7)), the responsivity (Equation (9)) and the NEP (Equation (12)), several material parameters and electrical parameters are required. The carrier mobility and carrier concentration for PCA A, PCA $B$ and the receiver shown in [14], henceforth termed PCA C, were measured by high resistance Hall measurements (Lakeshore 8400). The two wafers show nominally the same layer structure, except that the PCA A and PCA B are Be-doped while PCA C is C-doped. The mobility of the wafer of PCA A and $B$ has been found to $186 \mathrm{~cm}^{2} /$ Vs while that of PCA C was $775 \mathrm{~cm}^{2} / \mathrm{Vs}$. The carrier concentration has been determined to $n_{A, B}=8.6 \times 10^{12} \mathrm{~cm}^{-3}$ and $n_{C}=3.4 \times 10^{13} \mathrm{~cm}^{-3}$. We note that these values are only about 14 times (54 times) higher than the intrinsic carrier density, $n_{i}$, of InGaAs. The samples can therefore be considered as very well compensated. The sheet resistance of the wafer of PCA A and B was determined to $3.87 \times 10^{7} \Omega / \square$, and that of PCA C to $2.32 \times 10^{6} \Omega / \square$. We note that due to excellent compensation, these values may depend on the exact position on the wafer. The Hall data suggest that PCA C should be about 16 times more conductive than PCA A and PCA B. However, comparing nine resistive structures from the Be-doped wafer (including PCA A and B) to five structures from the C-doped wafer supports only a resistance ratio of a factor of 2.5. PCA A and B were processed from a piece close to the border of the wafer. While for PCA C the Hall resistance fits very well to the measured resistance of processed THz devices, the resistances of PCA A and B are about three to four times smaller than expected from the Hall sheet resistance. We therefore conclude that the Hall data can be considered correct for PCA C, while the Hall data for the Be-doped wafer differ from the values of the processed devices PCA A and B. Further, it is astonishing that Be-doping reduces the carrier mobility by a factor of four, despite nominally same doping levels and same layer structure. The carrier lifetime for the two wafers examined in this paper has been determined to $0.53 \mathrm{ps}$ and $0.52 \mathrm{ps}$ in [19]. The absorption of the samples was determined by monochromator measurements to $\approx 0.53 \pm 0.05$, corresponding to an absorption coefficient of $7600 / \mathrm{cm}$, assuming negligible reflection 
by the anti-reflection coating of the samples. For PCA C, we used a high numerical aperture lens with a focus spot size in the range of $2 w_{G}$. We therefore assume only small losses by reflection of the metallic finger structure, yielding $\eta_{o p t}^{C} \approx 0.50 \pm 0.07$. PCA A and PCA B were fiber-pigtailed. The coupling efficiency is therefore lower than for PCA C and estimated to be 0.5 for PCA A, and 0.6 for PCA B, by comparing the photocurrent of the packaged to the photocurrent of unpackaged devices that used the same focusing optics as PCA C. This yields an optical efficiency $\eta_{o p t}^{A} \approx 0.25 \pm 0.04$, $\eta_{\text {opt }}^{B} \approx 0.30 \pm 0.04$ for PCA A and B, respectively. The DC resistance $R_{P C}^{A}=16.66 \mathrm{k} \Omega, R_{P C}^{B}=12.8 \mathrm{k} \Omega$ for PCA A and B, respectively, was recorded under laser illumination by applying a bias of $0.2 \mathrm{~V}$. A comparison to the theoretical value in Equation (7) allows to calculate the biasing efficiency of PCA $C$ to $\eta_{\text {bias }} \approx 0.96$. Considering the fact that the Hall data and $\eta_{o p t}^{C}$ are prone to errors, this value is very close to the estimated values from theoretical considerations, that lie between 0.75 and 0.9 . For PCA A and B, however, $\eta_{\text {bias }}$ would be larger than 1 . This is in line with the fact that Hall data and measured resistances do not match and that the measured Hall mobility is about four times less for the Be-doped wafer (PCA A and B) than for the C-doped wafer (PCA C). If we assume the same mobility of $775 \mathrm{~cm}^{2} /(\mathrm{Vs})$ and same biasing efficiency $\eta_{\text {bias }}$ (same electrode structure and sample thickness) for PCA A, B and C, the optical coupling efficiency is 2.2 and 2.9 times lower for PCA A and B, respectively, as compared to PCA C. This compares well with optical losses by imperfect packaging and further supports the assumption that the Hall measurement underestimates the mobility of PCA A and B.

For the THz performance, the antenna impedance $Z_{A}(f)$ and device capacitance $C$ were calculated by EM simulations using CST microwave studio. As explained in Section 2, the effective terahertz efficiency $\eta_{T H z}^{e f f}$ was obtained from the experimentally obtained responsivity $S(f)$ for four different frequencies via linear fitting, as shown in Figure 4. The fitted values were then evaluated using Equation (10) in order to get four different values of $\eta_{T H z}^{\text {eff }}$ (one for each frequency). The four obtained values of $\eta_{T H z}^{e f f}$ were then averaged to get a final value given that they did not vary more than $20 \%$. The measurements for this experimental determination of $S(f)$ were done using only PCA B.

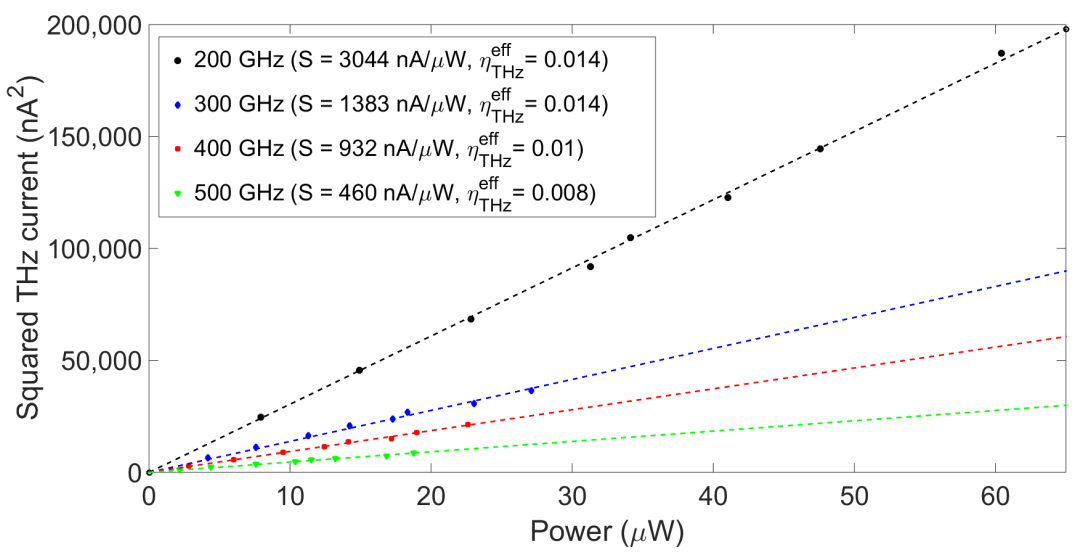

Figure 4. Squared detected THz current versus measured THz power for PCA B. The fitted responsivity $S(f)$ (shown in dashed lines) was equated to Equation (10) in order to obtain the effective $\mathrm{THz}$ effiency $\eta_{T H z}^{\text {eff }}$. The minimum THz power that could be measured was limited by the sensitivity of the PTB calibrated detector $(1 \mu \mathrm{W})$.

All the parameters required to calcuate $S(f)$ and $N E P_{T H z}(f)$, for the whole frequency range of interest, are summarized in Table 1. $Z_{A}(f)$ oscillates between the boundaries listed in the table. For the following calculations, the actual frequency-dependent, simulated $Z_{A}(f)$ is used. The averaged value of $\eta_{T H z}^{\text {eff }}=0.012 \pm 0.0024$ contains the (i) reflection loss of the silicon lens (30\%), (ii) reflections due to mismatch between the antenna impedance and the silicon wave impedance $\left(Z_{S i}=110 \Omega\right)$, (iii) mismatch between the $\mathrm{THz}$ spot size and the receiving area of the antenna, as well as (iv) differences between DC and AC parameters. The antenna radiation impedance oscillates around $80 \Omega$, 
slightly below the wave impedance of silicon. At a point of impedance-matching $\left(Z_{A}(f)=Z_{S i}\right)$, we estimate the total losses of (i)-(iii) to be in the range of a factor of $\eta_{T H z} \approx 0.4$. With the definition of $\eta_{T H z}^{e f f}=\eta_{T H z} \frac{\mu_{T H z}^{2}}{\mu_{D C}^{2}}$, the AC mobility and THz non-idealities are about five to six times worse than under DC conditions.

Table 1. PCA parameters for calculation of responsivity and noise-equivalent power (NEP). $I_{\text {noise }}$ is the measured current noise of the detectors while $I_{\text {therm }}$ is the ideal thermal noise floor.

\begin{tabular}{|c|c|c|c|c|c|c|c|c|}
\hline PCA & $Z_{A}(\Omega)$ & $C(\mathrm{fF})$ & $\eta_{T H z}^{e f f}$ & $\tau_{r e c}(\mathrm{fs})$ & $R_{P C}(\mathrm{k} \Omega)$ & $P_{L}(\mathrm{~mW})$ & $I_{\text {therm }}(\mathrm{pA} / \sqrt{\mathrm{Hz}})$ & $I_{\text {noise }}(\mathrm{pA} / \sqrt{\mathrm{Hz}})$ \\
\hline A & $\begin{array}{c}47.3-5 \mathrm{i}- \\
119.3+30.4 \mathrm{i}\end{array}$ & 3.6 & 0.012 & 530 & 16.66 & 25.3 & 0.993 & 2.59 \\
\hline B & $\begin{array}{c}47.3-5.0 \mathrm{i}- \\
119.3+30.4 \mathrm{i}\end{array}$ & 3.6 & 0.012 & 530 & 12.8 & 24.7 & 1.13 & 7.03 \\
\hline C & $\begin{array}{l}60.8+5.0 \mathrm{i}- \\
95.5+17.5 \mathrm{i}\end{array}$ & 3.6 & NA & 520 & 5.6 & 26 & 1.72 & 8.48 \\
\hline
\end{tabular}

The experimentally obtained responsivity, $S(f)=I_{r}^{2}(f) / P_{T H z}(f)$, and the calculated one using Equation (10) and the parameters from Table 1 are shown in Figure 5 for PCA A and B. The actual measured $\mathrm{THz}$ powers and currents used for the determination of the experimental responsivity can be found in Appendix A.

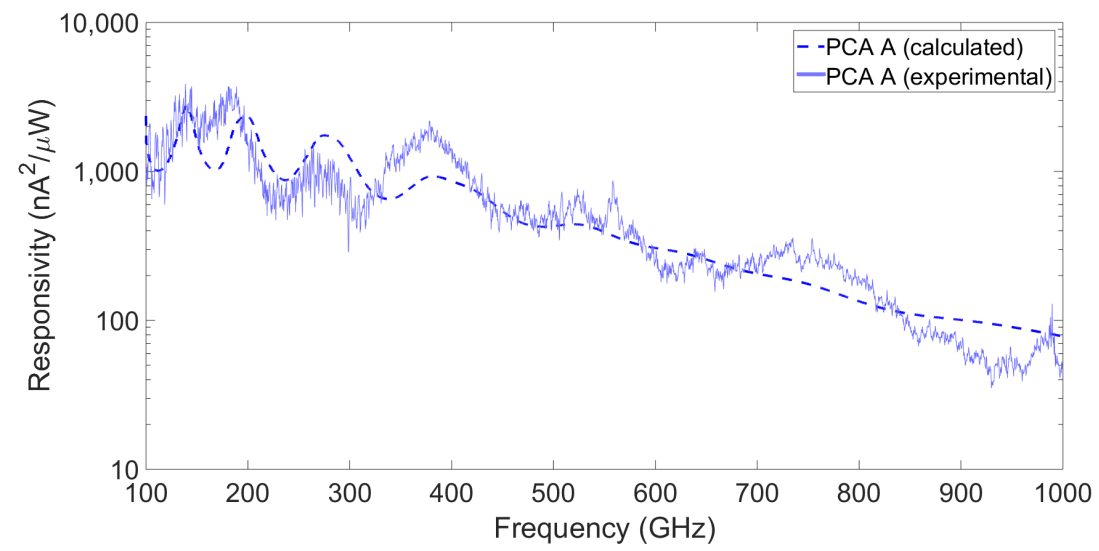

(a)

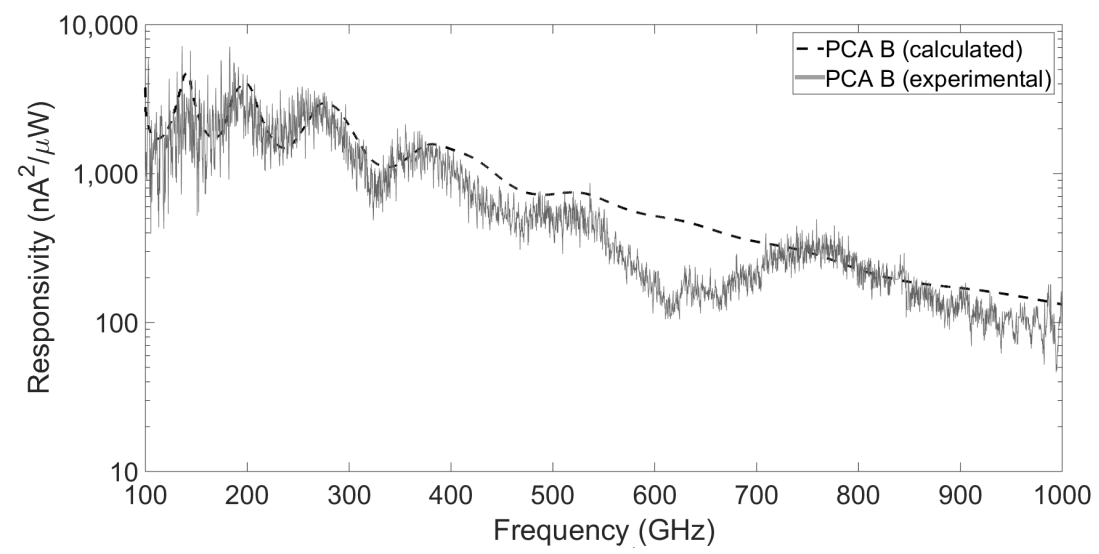

(b)

Figure 5. Comparison between the calculated and experimentally obtained responsivities of (a) PCA A (b) PCA B. $\eta_{T H z}^{\text {eff }}$ is assumed frequency-independent.

The agreement between the calculated and the experimentally obtained responsivity is excellent for both PCAs for the whole examined frequency range, using only one parameter extracted from 
the experimental data of PCA B, namely $\eta_{T H z}^{e f f}$. This shows that the theoretical model presented in Section 2 accurately describes the rectified current for a given incident $\mathrm{THz}$ power.

While a photoconductor is actually a mixer, it does not need any external $\mathrm{THz}$ local oscillator source as opposed to other $\mathrm{THz}$ mixer detectors like Schottky diodes, superconductor-insulator-superconductor (SIS) tunnel junctions, or hot electron-bolometers (HEBs). They can therefore be used as $\mathrm{THz}$ power detectors, however, with amplitude and phase sensitivity. For determination of the NEP as defined by Equation (11), the noise current density, $I_{\text {noise }}$, has been recorded with laser power but without any $\mathrm{THz}$ power incident on the receivers. It is summarized, together with the thermally limited noise current, $I_{\text {therm }}=\sqrt{4 k_{B} T / R_{P C}}$, in Table 1 . For all samples, the theoretical noise current is lower than the experimental values, by a factor of 2.6, 6.2 and 4.9 for PCA A, B, and C, respectively. The theoretical NEP represents the lowest limit in a perfect detection chain. In the experimental setup, however, further noise sources are present. Both the transimpedance amplifier (TIA) and the lock-in amplifier add noise. In [14], the noise floor of the same model of TIA and post detection circuit was determined to $1.45 \mathrm{pA} / \sqrt{\mathrm{Hz}}(1.9 \mathrm{pA}$ for a detection bandwidth of $1.67 \mathrm{~Hz}$ ). Further, incoupling of external noise sources by stray fields with frequency components at the modulation frequency increase the noise floor. Last but not least, the NEP calculation neglects laser noise which, in turn, modulates $R_{P C}$ and thus increases the noise floor. Therefore, the experimental NEPs of PCA A and B are by a factor of $2.6^{2}=6.8$ and $6.2^{2}=38.4$ higher than the thermal limit, in agreement with the experimental findings shown in Figure 6, where the calculated NEP assumed the thermally limited noise current and the calculated responsivity shown by the dashed lines in Figure 5 . The lowest measured NEP of $1.81 \mathrm{fW} / \mathrm{Hz}$ was achieved with PCA A at $188.8 \mathrm{GHz}$. For a detection bandwidth of $1 \mathrm{~Hz}$, this value is about five orders of magnitude smaller than the NEP of a Golay cell (direct detection NEP $\sim 150 \mathrm{pW} / \sqrt{\mathrm{Hz}}$ ) and about three orders of magnitude lower than values reported for Schottky diodes (direct detection NEP $\sim 1.5-20 \mathrm{pW} / \sqrt{\mathrm{Hz}}$ at $200 \mathrm{GHz}$ [20-22], where some of them are hollow waveguide-coupled devices with excellent noise shielding).

A comparison of the performance between the ErAs:InGaAs PCAs and other THz mixers (e.g., SIS junctions, Schottky diodes or HEBs) in terms of noise equivalent temperature difference (NETD) would require a different set of measurements, i.e., using non-coherent, broadband hot and cold loads as sources [23]. The goal here was a characterizatio of PCAs as coherent detectors.

Finally, we also estimate the lowest possible NEP for the three PCAs by allowing the AC transport to approximate the DC performance, considering a perfect $\mathrm{THz}$ coupling $\left(\eta_{T H z}^{\text {eff }}=1\right)$, and assuming a noise floor limited only by thermal noise. At $200 \mathrm{GHz}$, such ideal devices would result in NEPs of $4.2 \mathrm{aW} / \mathrm{Hz}, 3.3 \mathrm{aW} / \mathrm{Hz}$, and $3.3 \mathrm{aW} / \mathrm{Hz}$ for PCA A, B and C, respectively. For the rest of the frequency range the ideal peformance is shown in Figure 6.

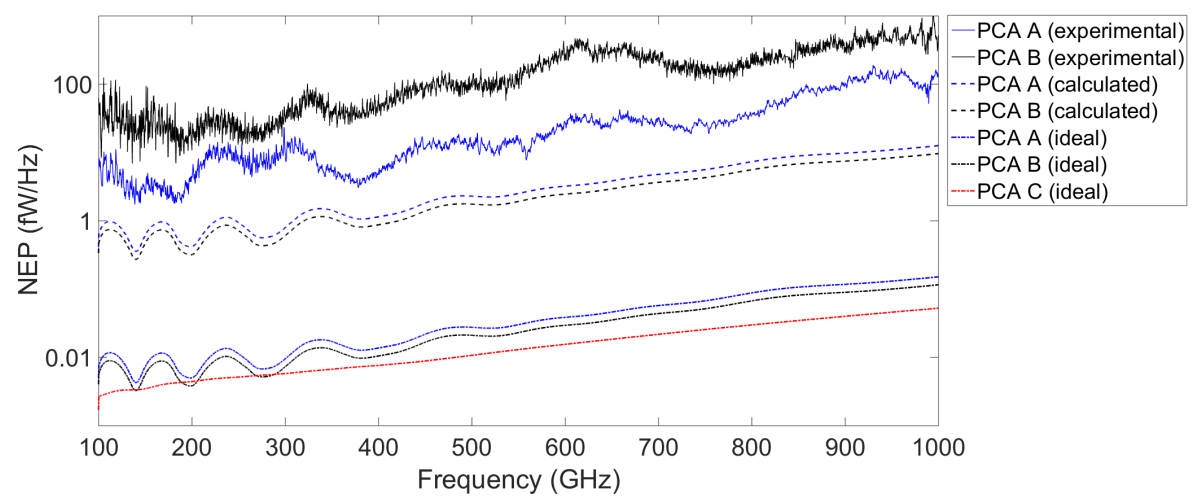

Figure 6. Comparison between the calculated $\left(\eta_{T H z}^{\text {eff }}=0.012, I_{\text {noise }}=I_{\text {therm }}\right)$, the ideal (with $\eta_{T H z}^{\text {eff }}=1$, $\left.I_{\text {noise }}=I_{\text {therm }}\right)$, and the experimentally obtained NEPs for PCA A and B. The figure also shows the ideal performance of PCA C. 


\section{Conclusions}

We have derived a theoretical model for the CW responsivity and NEP of PCAs and compared the model to experimental results from homodyne ErAs:InGaAs PCA detectors driven by $1550 \mathrm{~nm}$ lasers at room temperature. Only one fitting parameter was required, namely the $\mathrm{THz}$ coupling efficiency $\eta_{T H z}^{\text {eff }}$ in order to achieve agreement between the theoretical model and experimental results from $100 \mathrm{GHz}$ to $1 \mathrm{THz}$. The lowest NEP of $1.81 \mathrm{fW} / \mathrm{Hz}$, SI traceable by a PTB calibrated power detector, was achieved at $188.8 \mathrm{GHz}$. We identified a discrepancy between the expected and measured $\mathrm{THz}$ coupling efficiency that requires further studies. Ideal devices are expected to reach noise floors in the range of 3.3-4.2 aW/ Hz at $200 \mathrm{GHz}$.

Author Contributions: Conceptualization: S.P. and A.D.J.F.O.; Methodology: A.D.J.F.O.; Software: A.R.; Validation: S.P.; Formal Analysis: S.P., A.R.; Theory: S.P.; Investigation: A.D.J.F.O.; Resources: N.V., H.L. and A.C.G.; Data Curation: A.D.J.F.O.; Writing-Original Draft Preparation: A.D.J.F.O.; Writing—Review \& Editing: S.P., K.D., A.R.; Visualization: A.D.J.F.O.; Supervision: S.P., K.D.; Project Administration: S.P. and N.V.; Funding Acquisition: S.P.

Funding: This research was funded by the European Union Marie Curie Skłodowska Actions through the Innovative Training Network (ITN) with project number 675683 (CELTA), and by the European Starting Grant (ERC) with project number 713780 (Pho-T-Lyze).

Acknowledgments: The authors would like to acknowledge Andreas Steiger (from the PTB) for the advice given during the THz power measurements, Anselm Deninger (from TOPTICA photonics) for the organizational efforts leading to realization of paper, and Computer Simulation Technologies (CST) AG for the EM Simulation solver.

Conflicts of Interest: The authors declare no conflict of interest. The funding agencies had no role in the design of the study; in the collection, analysis, or interpretation of data; in the writing of the manuscript, and in the decision to publish the results.

\section{Appendix A. Measured Power and Currents}

This appendix shows the measured powers (Figure A1) and the measured rectified currents (Figure A2) used for the characterization of the responsivity and the NEP of PCA A and B. Figure A2 also shows a comparison with the rectified currents predicted by the model of Section 2. These were calculated using Equation (5), the parameters of Table 1, and the measured powers of Figure A1. The agreement between the two is excellent.

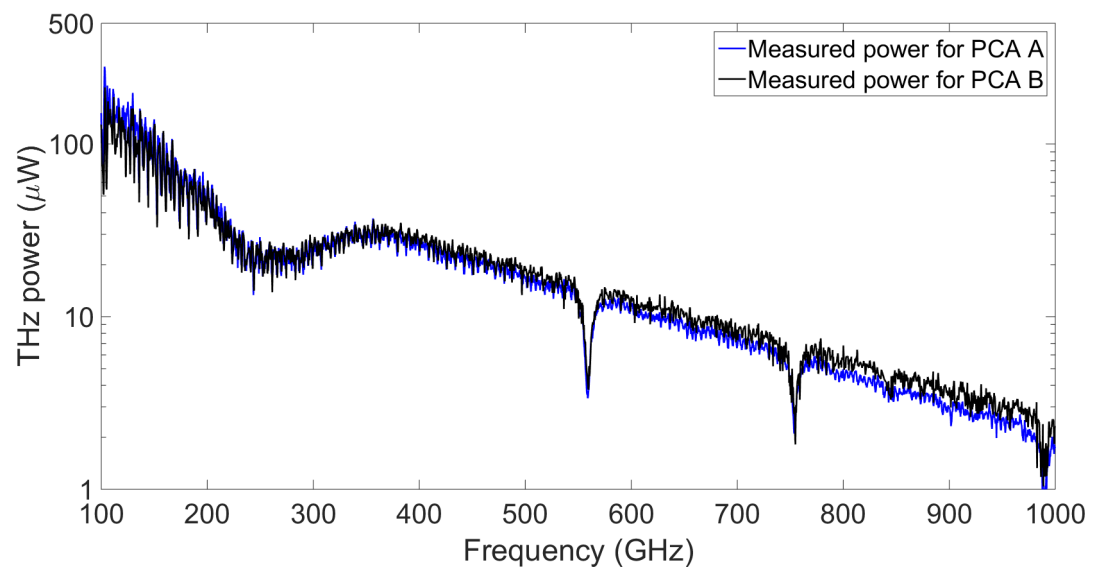

Figure A1. Measured THz powers emitted by the pin diode for the characterization of PCA A and B.

It is interesting to note that there is a slight difference between the higher frequency end of the two power measurements shown in Figure A2. This slight difference is likely caused by the different conditions in which both measurements were taken: The setup had to be rebuilt from scratch for the second measurement, the pyroelectric detector model used was different, and the laser power in the PIN diode was also marginally different (as mentioned in Section 3). On the other hand, the fact that 
the measured $\mathrm{THz}$ power is highly consistent under different measurements conditions, especially for the lower frequency end, gives confidence in the accuracy of the alignment.
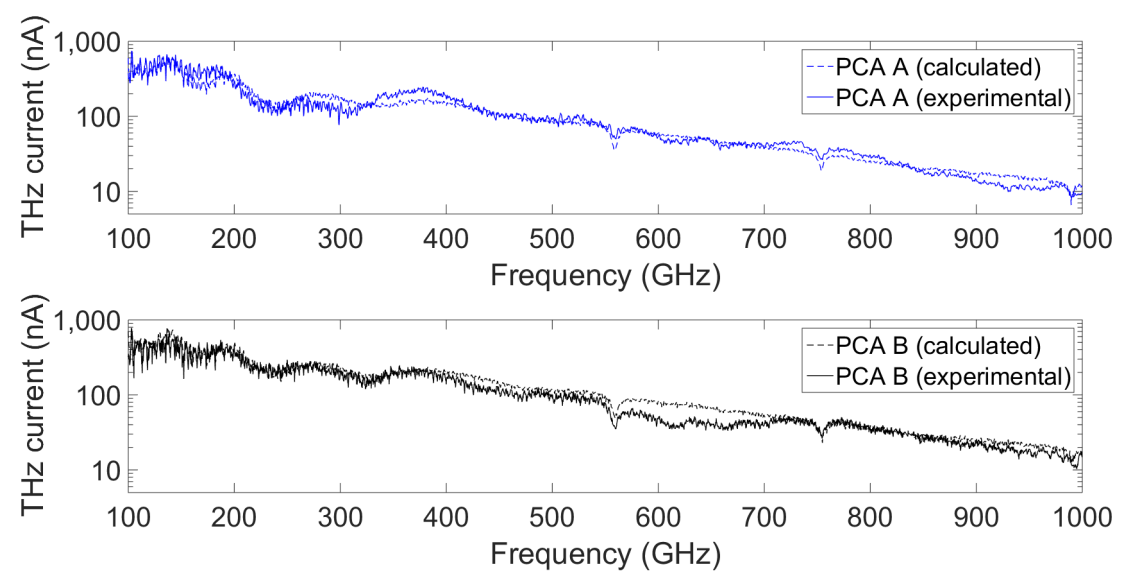

Figure A2. Comparison between the experimentally obtained and the calculated rectified current of PCA A and B.

\section{References}

1. Deninger, A.; Göbel, T.; Schönherr, D.; Kinder, A.; Roggenbuck, A.; Köberle, M.; Lison, F. Precisely tunable continuous-wave terahertz source with interferometric frequency control. Rev. Sci. Instrum. 2008, 79, 044702. [CrossRef] [PubMed]

2. Harter, T.; Weber, M.; Muehlbrandt, S.; Wolf, S.; Kemal, J.; Boes, F.; Nellen, S.; Goebel, T.; Giesekus, J.; Zwick, T.; et al. Wireless THz Communications Using Optoelectronic Techniques for Signal Generation and Coherent Reception. In Proceedings of the Conference on Lasers and Electro-Optics, San Jose, CA, USA, 14-19 May 2016.

3. Harter, T.; Adib, M.M.H.; Wolf, S.; Muehlbrandt, S.; Weber, M.; Blaicher, M.; Boes, F.; Massler, H.; Tessmann, A.; Nellen, S.; et al. Wireless Multi-Subcarrier THz Communications Using Mixing in a Photoconductor for Coherent Reception. In Proceedings of the IEEE Photonics Conference, Orlando, FL, USA, 1-5 October 2017.

4. Scheller, M.; Baaske, K.; Koch, M. Multifrequency continuous wave terahertz spectroscopy for absolute thickness determination. Appl. Phys. Lett. 2010, 96, 151112. [CrossRef]

5. Wilk, R.; Breitfeld, M.; Mikulics, M.; Koch, M. Continuous wave terahertz spectrometer as a noncontact thickness measuring device. Appl. Opt. 2008, 47, 3023-3026. [CrossRef] [PubMed]

6. Stanze, D.; Globisch, B.; Dietz, J.B.R.; Roehle, H.; Göbel, T.; Schell, M. Multilayer Thickness Determination Using Continuous Wave THz Spectroscopy. IEEE Trans. Terahertz Sci. Technol. 2014, 4, 696-701. [CrossRef]

7. Sartorius, B.; Schlak, M.; Stanze, D.; Roehle, H.; Künzel, H.; Schmidt, D.; Bach, H.-G.; Kunkel, R.; Schell, M. Continuous wave terahertz systems exploiting $1.5 \mu \mathrm{m}$ telecom technologies. Opt. Express 2009, 17, 15001-15007. [CrossRef] [PubMed]

8. Sukhotin, M.; Brown, E.R.; Gossard, A.C.; Driscoll, D.; Hanson, M.; Maker, P.; Muller, R. Photomixing and photoconductor measurements on ErAs/InGaAs at $1.55 \mu \mathrm{m}$. Appl. Phys. Lett. 2003, 82, 3116-3118. [CrossRef]

9. Göbel, T.; Stanze, D.; Globisch, B.; Dietz, J.B.R.; Roehle, H.; Schell, M. Telecom technology based continuous wave terahertz photomixing system with 105 decibel signal-to-noise ratio and 3.5 terahertz bandwidth. Opt. Lett. 2013, 38, 4197-4199. [CrossRef] [PubMed]

10. Kim, J.-Y.; Song, H.-J.; Yaita, M.; Hirata, A.; Ajito, K. CW-THz vector spectroscopy and imaging system based on 1.55- $\mu \mathrm{m}$ fiber-optics. Opt. Express 2014, 22, 1735-1741. [CrossRef] [PubMed]

11. Globisch, B.; Dietz, R.J.B.; Göbel, T.; Schell, M.; Bohmeyer, W.; Müller, R.; Steiger, A. Absolute terahertz power measurement of a time-domain spectroscopy system. Opt. Lett. 2015, 40, 3544-3547. [CrossRef] [PubMed] 
12. Garufo, A.; Carluccio, G.; Freeman, J.R.; Bacon, D.R.; Llombart, N.; Linfield, E.H.; Davies, A.G.; Neto, A. Norton Equivalent Circuit for Pulsed Photoconductive Antennas-Part II: Experimental Validation. IEEE Trans. Antennas Propag. 2018, 66, 1646-1659. [CrossRef]

13. Steiger, A.; Muller, R.; Remesal Oliva, A.; Deng, Y.; Sun, Q.; White, M.; Lehman, J. Terahertz Laser Power Measurement Comparison. IEEE Trans. Terahertz Sci. Technol. 2016, 6, 664-669.

14. Fernandez Olvera, A.D.J.; Lu, H.; Gossard, A.C.; Preu, S. Continuous-wave $1550 \mathrm{~nm}$ operated terahertz system using ErAs:In(Al)GaAs photoconductors with $52 \mathrm{~dB}$ dynamic range at $1 \mathrm{THz}$. Opt. Express 2017, 25, 29492-29500. [CrossRef]

15. Preu, S. A Unified Derivation of the Terahertz Spectra Generated by Photoconductors and Diodes. J. Infrared Milli Terahz Waves 2014, 35, 998-1010. [CrossRef]

16. Collin, R.E. Limitations of the Thevenin and Norton Equivalent Circuits for a Receiving Antenna. IEEE Trans. Antennas Propag. Mag. 2003, 45, 119-124. [CrossRef]

17. Garufo, A.; Carluccio, G.; Freeman, J.R.; Bacon, D.R.; Llombart, N.; Linfield, E.H.; Davies, A.G.; Neto, A. Norton Equivalent Circuit for Pulsed Photoconductive Antennas-Part I: Theoretical model. IEEE Trans. Antennas Propag. 2018, 66, 1635-1645. [CrossRef]

18. Müller-Landau, C.; Malzer, S.; Weber, H.B.; Döhler, G.H.; Winnerl, S.; Burke, P.; Gossard, A.C.; Preu, S. Terahertz generation with ballistic photodiodes under pulsed operation. Semicond. Sci. Technol. 2018, 33, 11. [CrossRef]

19. Suen, J.Y.; Krogen, P.R.; Preu, S.; Lu, H.; Gossard, A.C.; Driscoll, D.C.; Lubin, P.M. Measurement and modeling of ErAs: In0. 53Ga0. 47As nanocomposite photoconductivity for THz generation at $1.55 \mu \mathrm{m}$ pump wavelength. J. Appl. Phys. 2014, 116, 013703. [CrossRef]

20. Liu, L.; Hesler, J.L.; Weikle, R.M.; Wang, T.; Fay, P.; Xing, H. A 570-630 GHz frequency domain terahertz spectroscopy system based on a broadband quasi-optical zero bias schottky diode detector. Int. J. High Speed Electron. Syst. 2011, 20, 629-638. [CrossRef]

21. Semenov, A.; Cojocari, O.; Hübers, H.-W.; Song, F.; Klushin, A.; Müller, A.-S. Application of Zero-Bias Quasi-Optical Schottky-Diode Detectors for Monitoring Short-Pulse and Weak Terahertz Radiation. IEEE Electron Devices Lett. 2010, 31, 674-675. [CrossRef]

22. Hesler, J.L.; Crowe, T.W. Responsivity and Noise Measurements of Zero-Bias Schottky Diode Detectors. In Proceedings of the 18th International Symposium on Space Terahertz Technology, Pasadena, CA, USA, 21-23 March 2007; pp. 674-676.

23. Blundell, R.; Miller, R.E.; Gundlach, K.H. Understading Noise in SIS receivers. J. Infrared Milli Terahz Waves $1992,13,1$.

(C) 2019 by the authors. Licensee MDPI, Basel, Switzerland. This article is an open access article distributed under the terms and conditions of the Creative Commons Attribution (CC BY) license (http://creativecommons.org/licenses/by/4.0/). 